\title{
the Socially Stable Core in Structured Transferable Utility Games
}

Citation for published version (APA):

Herings, P. J. J., van der Laan, G., \& Talman, A. J. J. (2007). the Socially Stable Core in Structured Transferable Utility Games. Games and Economic Behavior, 59, 85-104.

https://doi.org/10.1016/j.geb.2006.04.008

Document status and date:

Published: 01/01/2007

DOI:

10.1016/j.geb.2006.04.008

Document Version:

Publisher's PDF, also known as Version of record

Document license:

Taverne

Please check the document version of this publication:

- A submitted manuscript is the version of the article upon submission and before peer-review. There can be important differences between the submitted version and the official published version of record.

People interested in the research are advised to contact the author for the final version of the publication, or visit the DOI to the publisher's website.

- The final author version and the galley proof are versions of the publication after peer review.

- The final published version features the final layout of the paper including the volume, issue and page numbers.

Link to publication

\footnotetext{
General rights rights.

- You may freely distribute the URL identifying the publication in the public portal. please follow below link for the End User Agreement:

www.umlib.nl/taverne-license

Take down policy

If you believe that this document breaches copyright please contact us at:

repository@maastrichtuniversity.nl

providing details and we will investigate your claim.
}

Copyright and moral rights for the publications made accessible in the public portal are retained by the authors and/or other copyright owners and it is a condition of accessing publications that users recognise and abide by the legal requirements associated with these

- Users may download and print one copy of any publication from the public portal for the purpose of private study or research.

- You may not further distribute the material or use it for any profit-making activity or commercial gain

If the publication is distributed under the terms of Article $25 \mathrm{fa}$ of the Dutch Copyright Act, indicated by the "Taverne" license above, 


\title{
The socially stable core in structured transferable utility games
}

\author{
P. Jean-Jacques Herings ${ }^{\mathrm{a}, *}$, Gerard van der Laan ${ }^{\mathrm{b}}$, Dolf Talman ${ }^{\mathrm{c}}$ \\ a Department of Economics, Maastricht University, PO Box 616, 6200 MD Maastricht, The Netherlands \\ ${ }^{\mathrm{b}}$ Department of Econometrics and Tinbergen Institute, Free University, De Boelelaan 1105, \\ 1081 HV Amsterdam, The Netherlands \\ c Department of Econometrics and Operations Research and CentER, Tilburg University, PO Box 90153, \\ 5000 LE Tilburg, The Netherlands
}

Received 21 May 2004

Available online 28 August 2006

\begin{abstract}
We consider socially structured transferable utility games. For every coalition the relative strength of a player is measured by a power function. The socially stable core consists of the socially and economically stable payoff vectors. It is non-empty if the game itself is socially stable. The socially stable core consists of a finite number of faces of the core. Generically, it consists of a finite number of payoff vectors. Convex TU-games have a non-empty socially stable core, irrespective of the underlying social structure. When the game is permutationally convex, the socially stable core is shown to be non-empty if the power vectors are permutationally consistent and is shown to contain a unique element if the power vectors are permutationally compatible. We demonstrate the usefulness of the concept of the socially stable core by applying it to structured hierarchy games, sequencing games and the distribution of water.
\end{abstract}

(C) 2006 Elsevier Inc. All rights reserved.

JEL classification: $\mathrm{C} 60 ; \mathrm{C} 70 ; \mathrm{D} 70$

Keywords: Transferable utility game; Social structure; Hierarchy; Socially stable core

\footnotetext{
* Corresponding author. Fax: +31433884878.

E-mail addresses: P.Herings@algec.unimaas.nl (P.J.-J. Herings), glaan@feweb.vu.nl (G. van der Laan), talman@uvt.nl (D. Talman).

URL: http://www.fdewb.unimaas.nl/algec/ (P.J.-J. Herings).
} 


\section{Introduction}

A situation in which a finite set of players can obtain certain payoffs by cooperation can be described by a cooperative game with transferable utility, or simply a TU-game. In a TU-game players only differ with respect to their position in the game. Examples of models in which players not only differ with respect to their position in the game, but also are part of some relational structure (which possibly affects the cooperation possibilities or payoff distributions) are games in coalition structure and games with limited communication structure. In games with coalition structure it is assumed that the set of players is partitioned into disjoint sets which represent social groups. For a particular player it is more easy to cooperate with players in his own group than to cooperate with players in other groups (see, e.g., Aumann and Drèze, 1974; Owen, 1977; Hart and Kurz, 1983; and Winter, 1989). In games with limited communication structure the edges of an undirected graph on the set of players represent binary communication links between the players such that players can cooperate only if they are connected (see, e.g. Myerson, 1977; Kalai et al., 1978; Owen, 1986; and Borm et al., 1992).

Another line of research in the field of cooperative games are situations in which the players are part of some hierarchical structure such as games with a permission structure. In such games it is assumed that players in a TU-game are part of a hierarchical organization in which there are players that need permission from other players before they are allowed to cooperate within a coalition, see for instance Gilles et al. (1992) and van den Brink and Gilles (1996). Related is also the model of Faigle and Kern (1992) who consider feasible rankings of the players. Demange (2004) provides a rationale for the fact that a hierarchical structure is a widespread organizational form in many areas.

In this paper we consider TU-games with an exogenously given social structure on any subset of the players, for instance a network, a hierarchical ordering or some dominance relation. We assume that for every coalition the underlying social structure is represented by a power vector, whose components reflect the strengths of the individual members of the coalition within the social structure. From the literature several methods are known to measure the power of the individual members. The power vector of a coalition is completely determined by the social structure along which the coalition is organized. More powerful players are able to increase their payoffs at the expense of less powerful players as far as the less powerful players don't have the possibility to obtain their payoffs within alternative internal organizations in which they are more powerful. A similar approach has been followed in Piccione and Rubinstein (2006), who consider an exchange economy in which the exogenous distribution of initial endowments has been replaced by an exogenously given linear ordering of the agents reflecting their strengths. In their economy, a player can appropriate goods belonging to a player he dominates in the linear order.

The concept of socially stable core has been introduced in Herings et al. (2003) within the more general framework of structured games with non-transferable utility. For a payoff vector to be in the socially stable core, there should be neither incentives to deviate from an economic point of view, nor from a social one. A payoff vector is economically stable if it is feasible and undominated, i.e., when the payoff vector is in the superadditive cover core of the game, and therefore in the core whenever the game is superadditive. No player has an incentive to deviate from a core payoff vector from an economic point of view. Socially motivated deviations do not occur when all individuals are equally powerful at the proposed payoff vector. This is formalized by considering the power vectors of all coalitions that could realize the proposed payoff vector for its members. If there is a weighted sum of these power vectors that gives all individuals the 
same power, then individuals are said to be equally powerful at the proposed payoff and the payoff vector is called socially stable. The socially stable core consists of all payoff vectors that are both economically and socially stable. In Herings et al. (2003), the property of social stability for a socially structured non-transferable utility game has been defined, and games satisfying this property are referred to as socially stable NTU-games. It has been shown that a socially stable game always has a non-empty socially stable core.

In this paper we consider the class of socially structured TU-games. We show that any convex game has a non-empty socially stable core. When the game is permutationally convex for some permutation, the socially stable core is shown to contain the corresponding marginal vector if the power vectors are permutationally consistent with respect to this permutation. When the game is permutationally convex and the power vectors are permutationally compatible for some permutation, the corresponding marginal vector is the unique element of the socially stable core. In general, the socially stable core consists of a finite union of faces of the set of economically stable payoff vectors. Moreover, it is proved that, generically, the socially stable core consists of a finite number of elements only. We demonstrate the usefulness of the concept of the socially stable core by applying it to structured hierarchy games, in which the underlying social structure of the whole set of players is a hierarchy and the social structure of any subset of players is induced by it. For power vectors representing this hierarchy, we prove that the socially stable core consists of only one element, if the game is superadditive. Finally, we consider some applications in which the hierarchy on the players is a linear order that arises from the characteristics of the economic situation. These applications concern sequencing games and the water distribution problem.

The structure of the paper is as follows. Section 2 gives some preliminaries. In Section 3 socially structured transferable utility games are introduced as well as the associated solution concept of the socially stable core. In Section 4 the main theorems are presented. Section 5 studies structured hierarchy games, and Section 6 sequencing games and the water distribution problem. Section 7 concludes.

\section{Preliminaries}

A situation in which a finite set of players can obtain certain payoffs by cooperation can be described by a cooperative game with transferable utility, or simply a TU-game, being a pair $(N, v)$, with $N=\{1,2, \ldots, n\}$ a finite set of $n$ players and $v: 2^{N} \rightarrow \mathbb{R}$ a characteristic function assigning to any coalition $S \subseteq N$ of players a real number $v(S)$ as the worth of coalition $S$ with $v(\emptyset)=0$, i.e., the members of coalition $S$ can obtain a total payoff of $v(S)$ by agreeing to cooperate. In this paper we assume that $N$ is a fixed set of players. We therefore denote a game $(N, v)$ shortly by its characteristic function $v$. A TU-game $v$ is superadditive if $v(S \cup T) \geqslant$ $v(S)+v(T)$ for any pair of subsets $S, T \subseteq N$ such that $S \cap T=\emptyset$. Further, a TU-game $v$ is convex if $v(S \cup T)+v(S \cap T) \geqslant v(S)+v(T)$ for all $S, T \subseteq N$.

A payoff vector is a vector $x \in \mathbb{R}^{n}$ assigning payoff $x_{i}$ to player $i \in N$. For a vector $y \in \mathbb{R}^{n}$, $y(S)=\sum_{i \in S} y_{i}$ denotes the sum over $S$ of the components of $y$. A solution $F$ assigns a set $F(v) \subset \mathbb{R}^{n}$ of payoff vectors to every TU-game $v$. A well-known set-valued solution is the core, assigning to every game $v$ the (possibly empty) set

$$
C(v)=\left\{x \in \mathbb{R}^{n} \mid x(N)=v(N) \text { and } x(S) \geqslant v(S), \text { for all } S \subseteq N\right\} .
$$

It is well known that the core of a TU-game $v$ is non-empty if and only if $v$ is balanced (see Bondareva, 1963). 
A more general notion of core is obtained by considering payoff configurations. The concept of payoff configuration was used by Aumann and Maschler (1964) when introducing the notion of the bargaining set, see also e.g. Friedman (1989) or Owen (1995). A payoff configuration of a TU-game $v$ is a pair $(x, \Pi)$, where $x$ is a payoff vector and $\Pi$ a partition $\left\{S_{1}, \ldots, S_{m}\right\}$ of $N$, satisfying $x\left(S_{j}\right)=v\left(S_{j}\right)$, for all $j=1, \ldots, m$. Let $P C$ be the set of all payoff configurations. A payoff configuration $(x, \Pi)$ is undominated if $x(S) \geqslant v(S)$ for all $S \subseteq N$. We now may define the superadditive cover as the set of all payoff vectors of undominated payoff configurations. Denoting this set as $\widetilde{C}(v)$, we have that

$$
\widetilde{C}(v)=\left\{x \in \mathbb{R}^{n} \mid \exists \Pi \text { s.t. }(x, \Pi) \in P C \text { and } x(S) \geqslant v(S) \text {, for all } S \subseteq N\right\} .
$$

Notice that $\widetilde{C}(v)=C(\tilde{v})$, where $\tilde{v}$ is the characteristic function given by the superadditive cover of $v$,

$$
\tilde{v}(S)=\max _{\left.\Pi \in \Pi\right|_{S}} \sum_{T \in \Pi} v(T),
$$

with $\left.\Pi\right|_{S}$ the collection of all partitions of $S$. The set of payoff vectors of undominated payoff configurations is equal to the core of its superadditive cover. When the game $v$ is superadditive and thus $\tilde{v}=v$, it holds that $\widetilde{C}(v)=C(\tilde{v})=C(v)$. It is for this reason that the results carry over to this more general core notion.

Aumann and Drèze (1974) take a fixed partition structure, say $\left.B \in \Pi\right|_{N}$, and define the core under partition structure $B$, denoted by $C(v, B)$, as the set of undominated payoff vectors satisfying $x(S)=v(S)$ for all $S \in B$. When $\tilde{v}(N)=\sum_{S \in B} v(S)$, it holds that $C(v, B)=\widetilde{C}(v)=C(\tilde{v})$. For a payoff vector $x$ in $\widetilde{C}(v)$ and for any partition $\left.B \in \Pi\right|_{N}$ satisfying $\sum_{S \in B} v(S)=\tilde{v}(N)$ we have that $x(S)=v(S)$ for all $S \in B$.

An important point-valued solution is the Shapley value. This value can be defined in several ways, for instance as a weighted sum of the so-called marginal contributions (see Shapley, 1953) or as an equal distribution of the so-called Harsanyi dividends of coalitions (see Harsanyi, 1959) among the players in the coalitions. Because of reasons later on in this paper, we use here the concept of marginal vector to define the Shapley value. For a permutation $\pi: N \rightarrow N$, assigning rank number $\pi(i) \in N$ to any player $i \in N$, define $\pi^{i}=\{j \in N \mid \pi(j) \leqslant \pi(i)\}$, i.e., $\pi^{i}$ is the set of all players with rank number at most equal to the rank number of $i$, including $i$ himself. Then the marginal value vector $m^{\pi}(v) \in \mathbb{R}^{n}$ of game $v$ and permutation $\pi$ is given by

$$
m_{i}^{\pi}(v)=v\left(\pi^{i}\right)-v\left(\pi^{i} \backslash\{i\}\right), \quad i \in N,
$$

and thus assigns to player $i$ his marginal contribution to the worth of the coalition consisting of all his predecessors in $\pi$. The Shapley value is equal to the average of the marginal value vectors over all permutations. When $v$ is convex, the core of $v$ is equal to the convex hull of all marginal value vectors and thus the Shapley value is in the core.

A game $v$ is permutationally convex, see Granot and Huberman (1982), if there exists a permutation $\pi$ such that for all $1 \leqslant j<k<n$ it holds that $\max \left[v(S), v\left(\pi^{j} \cup S\right)-v\left(\pi^{j}\right)\right] \leqslant$ $v\left(\pi^{k} \cup S\right)-v\left(\pi^{k}\right)$ for all $S \subset N \backslash \pi^{k}$. When a game $v$ is permutationally convex with respect to the permutation $\pi$, it holds that the corresponding marginal vector $m^{\pi}(v)$ is in the core and, hence, the core is non-empty.

\section{Structured TU-games}

Following Herings et al. (2003) for games with non-transferable utility, we assume in this paper that in a transferable utility game any subset of agents, including the grand coalition, is 
internally organized according to some social structure. A social structure could be a communication network (e.g., see Myerson, 1977), a hierarchy with a principal at the top and a function allocating to any of the other players a unique superior such that from each such player there is a unique path to the principal, or some other more general dominance relation. On any proper coalition, the social structure could be completely induced by the social structure on the grand coalition, as is often the case in models of network formation (e.g., see Jackson and Wolinsky, 1996), but it is also allowed that the social structure on a coalition is not induced by the social structure on the grand coalition but instead for instance is the most efficient internal organization for the coalition itself. In this paper we assume that the social structures on all the coalitions are given exogenously and that the characteristic function $v$ assigns to any coalition $S$ the value $v(S)$ of the coalition $S$ when organized according to its given social structure.

We assume that for every coalition $S$ there is a power vector $p(S)$ that represents the strength of the position of every player $i \in S$ within the underlying social structure of coalition $S$. In case the social structure is given by a directed or undirected graph on the set of players, we may use one of the power measures known from the literature. A well-known power measure is the degree or score measure, see e.g. Rubenstein (1980). According to this measure the power of a node in a given graph is equal to its (out)degree, being the number of edges in the graph to which the node is incident or in case of a directed graph the number of edges leaving the node. Other power measures for directed graphs have been proposed in for instance van den Brink and Gilles (2000) and Herings et al. (2005). In the latter paper, the positional power of a player in a directed graph takes into account the power of its successors and in a hierarchy the resulting power vector has the property that every player has more power than any of his successors. In case of undirected graphs, any of the so-called centrality measures may be used as a way to measure the power of a player.

A structured TU-game can therefore be described by a finite set of players, $N=\{1, \ldots, n\}$, a characteristic function $v: 2^{N} \rightarrow \mathbb{R}$, assigning to any coalition $S$ its value $v(S)$, and a social structure on every coalition, represented by a power function $p: \mathcal{N} \rightarrow \mathbb{R}^{n}$, assigning to every coalition $S$ the power vector $p(S)$ of its players within the underlying social structure of $S$, where $\mathcal{N}=2^{N} \backslash\{\emptyset\}$ is the collection of all non-empty subsets of $N$. It is assumed that both the characteristic function and the social structure (or equivalently its corresponding power vector) are exogenously given. For mathematical reasons we take the power vector $p(S)$ in $\mathbb{R}^{n}$ and not in $\mathbb{R}^{|S|}$. For a power vector $p(S)$ of coalition $S$ it holds that $p(S)$ is a nonnegative vector in $\mathbb{R}^{n}$, $p_{i}(S)=0$ for any $i$ not in $S$, and $p_{i}(S)>0$ for at least one player $i$ in $S$. We now have the following definition of a structured TU-game (STG).

Definition 3.1 (Structured TU-Game). A structured $T U$-game is given by the triple $\Gamma=(N, v, p)$ with $N$ a finite set of players, $v$ a characteristic function, and $p$ a power function.

In a structured TU-game we are interested in payoff vectors that are socially and economically stable. If for some payoff vector $x \in \mathbb{R}^{n}$ and coalition $S \in \mathcal{N}$ it holds that $x(S) \leqslant v(S)$, then coalition $S$ can obtain value $x(S)$ without cooperating with players outside $S$ and we say that coalition $S$ sustains $x$. If within coalition $S$ an individual at payoff vector $x$, sustained by $S$, has more power than some of the other individuals in $S$ and $x$ cannot be sustained by any other coalition, then this individual is able to increase his payoff at the expense of these other individuals. The payoff vector $x$ is in that case not socially stable. This process can only be stopped by a threat of some of the other individuals to form another coalition in which they have more power and can guarantee their coalition members at least the same payoff as before. 
A payoff vector $x$ is called socially stable if nonnegative real numbers or weights can be assigned to the coalitions $S$ sustaining $x$ in such a way that the weighted total power of every agent is equal to 1 and thus each agent has the same power. In the sequel, for $S \in \mathcal{N}$, let $e^{S} \in \mathbb{R}^{n}$ denote the vector given by $e_{i}^{S}=1$ when $i \in S$ and $e_{i}^{S}=0$ otherwise.

Definition 3.2 (Socially Stable Payoff). For a structured TU-game $\Gamma=(N, v, p)$, a payoff vector $x \in \mathbb{R}^{n}$ is socially stable if the system of equations

$$
\sum_{\{S \mid x(S) \leqslant v(S)\}} \lambda_{S} p(S)=e^{N}
$$

has a nonnegative solution.

Notice that a payoff vector $x$ satisfying $x(N) \leqslant v(N)$ is socially stable whenever $p(N)=e^{N}$. The next example shows that this may not hold when $p(N) \neq e^{N}$. In this example and also in later examples we often use, for ease of notation, as argument of $v$ and $p$ the elements of $S$ instead of the set $S$ itself.

Example 3.3. Take $N=\{1,2,3\}, v(1)=v(2)=v(3)=0, v(1,2)=4, v(1,3)=v(2,3)=2$ and $v(1,2,3)=5$, and $p(i)=e^{\{i\}}$ for $i=1,2,3, p(1,2)=(2,1,0)^{\top}, p(1,3)=(2,0,1)^{\top}, p(2,3)=$ $(0,1,2)^{\top}$ and $p(N)=(3,2,1)^{\top}$. Consider the payoff vector $x=(2,2,1)^{\top}$. Then $x(S) \leqslant v(S)$ for $S=\{1,2\}$ and $S=N$. Clearly, $e^{N}$ is not a nonnegative combination of $p(1,2)$ and $p(N)$, so $x$ is not socially stable. Observe that for any $S$ sustaining this $x$ it holds that $p_{1}(S)>p_{2}(S)>$ $p_{3}(S)$. When we increase at $x$ the payoff to player 1 at the expense of for instance player 2 until $y=(3,1,1)^{\top}$, then $y(S) \leqslant v(S)$ for $S=\{1,2\}, S=\{2,3\}$ and $S=N$. Now $e^{N}$ is a nonnegative linear combination of $p(1,2), p(2,3)$ and $p(N)$ and thus $y$ is socially stable. In fact $e^{N}$ is a nonnegative linear combination of only $p(1,2)$ and $p(2,3)$. As a result any payoff vector $x$ satisfying $x(S) \leqslant v(S)$ for $S=\{1,2\}$ and $S=\{2,3\}$ is socially stable, for instance $x=(4,0,2)^{\top}$.

In the sequel it will be useful to define stability of a collection of coalitions without reference to a particular payoff vector.

Definition 3.4 (Stable Collection of Coalitions). A collection of coalitions in $\mathcal{N},\left\{S_{1}, \ldots, S_{k}\right\}$, is stable if the system of equations

$$
\sum_{j=1}^{k} \lambda_{j} p\left(S_{j}\right)=e^{N}
$$

has a nonnegative solution. A stable collection of coalitions in $\mathcal{N}$ is minimal if no proper subset of it is stable.

A socially stable payoff vector is therefore a payoff vector that can be achieved by every element of some stable collection of coalitions. Observe that stability of a collection of coalitions reduces to balancedness of the collection when for every $S \in \mathcal{N}$ it holds that $p(S)=e^{S}$. In this case every member of a coalition has the same power. This might happen when for example the social structure of the grand coalition is a network connecting each pair of agents. In this way stability can be seen as a generalization of balancedness. In the remainder of this paper we denote the particular case of balancedness by $p=e$. 
A socially stable payoff vector may not be feasible. A payoff vector $x$ is said to be feasible if $x(N) \leqslant \tilde{v}(N)$, i.e., when the total payoff can be attained by cooperating according to some partition of the grand coalition. In Example 3.3 the socially stable vector $(4,0,2)^{\top}$ is not feasible, because $x(N)=6>5=\tilde{v}(N)=v(N)$ (because the game is superadditive). Furthermore, social stability of a payoff vector $x$ does not imply that $x$ is undominated, i.e., there may exist an $S \in \mathcal{N}$ and $y \in \mathbb{R}^{n}$ satisfying $y(S) \leqslant v(S)$ and $y_{i}>x_{i}$ for all $i \in S$. A payoff vector that is both feasible and undominated is called economically stable.

Definition 3.5 (Economically Stable Payoff). For a structured TU-game $\Gamma=(N, v, p)$, a payoff vector $x \in \mathbb{R}^{n}$ is economically stable if $x(N)=\tilde{v}(N)$ and $x(S) \geqslant v(S)$ for all $S \in \mathcal{N}$.

The definition says that a payoff vector $x$ is economically stable if and only if $x$ is in the superadditive cover core $\widetilde{C}(v)$ of $v$. The set of all socially and economically stable payoff vectors is called the socially stable core of the game.

Definition 3.6 (Socially Stable Core). The socially stable core of a structured TU-game $\Gamma=$ $(N, v, p)$, denoted $S C(v, p)$, consists of the set of socially and economically stable payoff vectors of $\Gamma$.

A payoff vector $x$ therefore lies in the socially stable core if and only if $x$ is feasible and undominated (economic stability) and can be sustained by a socially stable collection of coalitions (social stability). We want to stress that feasibility requires that $x(N) \leqslant \tilde{v}(N)$, but that sustainability is defined with respect to $v$. We conclude this section with two examples. The first example shows that a core element does not need to be socially stable.

Example 3.7. Consider the game $(N, v, p)$ with $N=\{1,2,3\}, v(1)=v(2)=v(3)=0$, $v(1,2)=1, v(1,3)=2, v(2,3)=3$, and $v(N)=6$. The social structure on $N$ is given by the hierarchy such that agent 1 dominates agent 3 and agent 3 dominates agent 2, e.g. $p_{1}(S)=3$ when $1 \in S, p_{2}(S)=1$ when $2 \in S$, and $p_{3}(S)=2$ when $3 \in S$. Clearly, $v$ is convex and therefore superadditive and thus $\tilde{v}(N)=v(N)$. The core is given by

$$
C(v)=\left\{x \in \mathbb{R}_{+}^{3} \mid x_{1}+x_{2}+x_{3}=6,0 \leqslant x_{1} \leqslant 3,0 \leqslant x_{2} \leqslant 4,0 \leqslant x_{3} \leqslant 5\right\} .
$$

Take any payoff vector $x$ in the relative interior of $C(v)$. Such a vector can only be sustained by the grand coalition $N$. Since $p^{N} \neq e^{N}$, the collection $\{N\}$ is not stable and therefore $x$ is not an element of the socially stable core. As can easily be seen, the only socially stable element in the core is $(3,0,3)^{\top}$, sustained by the stable collection $\{\{2\},\{2,3\}, N\}$. In this socially stable core element the weakest agent, agent 2 , gets his own value, the middleman, agent 3 , gets his marginal contribution when he joins the weakest agent, and the strongest agent, agent 1 , gets his marginal contribution when he joins the coalition of the weakest agent and the middleman.

The next example considers a game with four players, which is not superadditive. In particular it stresses the difference between feasibility and sustainability by the grand coalition $N$.

Example 3.8. Take $(N, v, p)$ with $N=\{1,2,3,4\}, v(1,2)=v(3,4)=2, v(S)=2$ when $|S|=3$, $v(N)=3$, and $v(S)=0$ otherwise; and $p(1,2)=(2,1,0,0)^{\top}, p(3,4)=(0,0,1,2)^{\top}$ and 
$p(S)=e^{S}$ for all other $S$. Clearly $\tilde{v}(N)=4$ and the set of undominated feasible payoff vectors is given by

$$
\widetilde{C}(v)=C(\tilde{v})=\left\{x \in \mathbb{R}_{+}^{4} \mid x_{1}+x_{2}=2, x_{3}+x_{4}=2\right\} .
$$

Now take any strictly positive vector $x \in \widetilde{C}(v)$. Then $x(S)>v(S)$ for all $S \neq\{1,2\},\{3,4\}$. Since $e^{N}$ cannot be written as a nonnegative linear combination of $p(1,2)$ and $p(3,4), x$ is not socially stable. In this case, the requirement of social stability removes all strictly positive vectors from $\widetilde{C}(v)$. It then easily follows that the unique element of $S C(v, p)$ is the payoff vector $x=(2,0,0,2)^{\top}$. For this payoff vector it holds that $x(S) \leqslant v(S)$ when $S$ is $\{2\},\{3\},\{1,2\}$ and $\{3,4\}$. Since $e^{N}$ is a nonnegative linear combination of $p(1,2), p(3,4), p(2)$ and $p(3)$, it holds that $x$ is sustained by the stable collection $\{\{2\},\{3\},\{1,2\},\{3,4\}\}$ and thus $x \in S C(v, p)$. Observe that for instance $x=(0,2,0,2)^{\top}$ is not socially stable, because $p_{1}(1,2)>p_{2}(1,2)$.

Notice that no $x \in \widetilde{C}(v)$ is socially stable although $p(N)=e^{N}$. The reason is that for each $x \in \widetilde{C}(v)$ it holds that $x(N)=\tilde{v}(N)>v(N)$ and thus $x$ is not sustained by $N$. To obtain total payoff equal to $4, N$ has to split itself in $\{1,2\}$ and $\{3,4\}$, so $x$ is sustained by these two coalitions. However, as shown above, the collection of these two coalitions is not socially stable.

\section{Non-emptiness of the socially stable core}

In this section we give sufficient conditions for the non-emptiness of the socially stable core

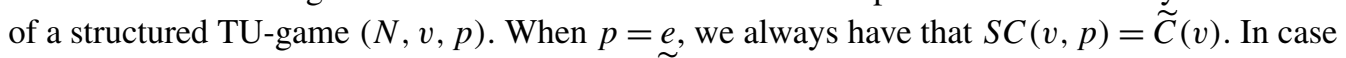
$v$ is also superadditive and thus $S C(v, p)=\widetilde{C}(v)=C(v)$, we know from Bondareva (1963) that the socially stable core is non-empty if and only if the game is balanced. However, for an arbitrary power function $p$ and arbitrary game $v$, the socially stable core is a subset of the superadditive cover core and might be empty even if the game is superadditive and balanced. The balancedness condition for superadditive TU-games is not sufficient for the non-emptiness of the socially stable core when $p \neq e$. The next definition of social stability of the game has been given in the NTU-context in Herings et al. (2003).

Definition 4.1 (Socially Stable Game). A structured TU-game $\Gamma=(N, v, p)$ is socially stable if every socially stable payoff $x$ of $\Gamma$ is feasible.

It should be observed that this social stability condition reduces to the usual balancedness condition of a TU-game when $p=e$ and the game is superadditive. We now have the following theorem.

Theorem 4.2. A structured $T U$-game $\Gamma=(N, v, p)$ has a non-empty socially stable core if $\Gamma$ is socially stable.

Proof. The proof follows from a more general theorem for socially structured NTU-games given in Herings et al. (2003).

The theorem above requires feasibility to be shown for any socially stable payoff vector. This may be a demanding task. On the other hand, social stability is sufficient but not necessary. The next theorem says that for every given power function a convex game has a non-empty socially stable core. Observe that the proof holds for any game whose core contains all marginal vectors. However, this does not generalize the result, because a game is convex iff the core contains 
all marginal vectors, see e.g. Ichiishi (1981). Observe that convexity implies superadditivity, so $x(N)=v(N)$ for any undominated payoff vector.

Theorem 4.3. If $v$ is a convex game, then for every power function $p$ the structured TU-game $\Gamma=(N, v, p)$ has a non-empty socially stable core.

Proof. To prove non-emptiness, we first construct for a given power function $p$ a stable collection of coalitions.

Step 1. Set $k=1, S_{k}=N, q^{k}=e^{N}$, and $r_{k}=n$. Go to Step 2 .

Step 2. Define $T_{k}=\left\{j \in S_{k} \mid p_{j}\left(S_{k}\right) / q_{j}^{k}=\max _{h \in S_{k}} p_{h}\left(S_{k}\right) / q_{h}^{k}\right\}$ and $t_{k}=\left|T_{k}\right|$. Define $\lambda_{k}=$ $\left(\max _{h \in S_{k}} p_{h}\left(S_{k}\right) / q_{h}^{k}\right)^{-1}$. Go to Step 3 .

Step 3. For $j \in T_{k}$, define $\pi(j) \in N$ such that $\left\{\pi(j) \mid j \in T_{k}\right\}=\left\{r_{k}, r_{k}-1, \ldots, r_{k}-t_{k}+1\right\}$. If $r_{k}=t_{k}$, define $k^{*}=k$ and stop the procedure; otherwise set $k=k+1$ and go to Step 4 .

Step 4. Set $S_{k}=S_{k-1} \backslash T_{k-1}, q^{k}=q^{k-1}-\lambda_{k} p\left(S_{k-1}\right)$ and $r_{k}=r_{k-1}-t_{k-1}=\left|S_{k}\right|>0$. Return to Step 2.

By construction we have that the collection $\left\{S_{1}, S_{2}, \ldots, S_{k^{*}}\right\}$ is a stable collection of coalitions and that $\pi=(\pi(1), \pi(2), \ldots, \pi(n))$ is a permutation of the elements of $N$ such that for any $k=1, \ldots, k^{*}$ it holds that

$$
S_{k}=\left\{\pi(1), \pi(2), \ldots, \pi\left(\ell_{k}\right)\right\}, \quad \text { with } \ell_{k}=\sum_{h=k}^{k^{*}} t_{h} .
$$

Next take the payoff vector $x$ equal to the marginal vector $m^{\pi}(v)$. Then it follows for any $k=$ $1, \ldots, k^{*}$ that

$$
\sum_{j \in S_{k}} x_{j}=\sum_{j=1}^{\ell_{k}}\left(v\left(\pi^{j}\right)-v\left(\pi^{j} \backslash\{j\}\right)\right)=v\left(\pi^{\ell_{k}}\right)=v\left(S_{k}\right) .
$$

By construction of the sets $S_{k}$ it follows that $x$ is socially stable. Moreover, since $v$ is convex we also have that $x \in C(v)$. Hence $x \in S C(v, p)$.

Observe that the marginal vector constructed in the proof is unique if and only if $k^{*}=n$ and thus $\left|T_{k}\right|=1$ for all $k$. When for some $k, T_{k}$ contains multiple players, we can take any order of the players within $T_{k}$ in Step 3. So, in general there are $\Pi_{k}\left|T_{k}\right|$ different permutations satisfying the conditions and inducing a marginal vector in $S C(v, p)$. We, however, remark that different permutations may induce the same marginal vector.

The assumptions in Theorems 4.2 and 4.3 are independent, i.e., socially stability of $\Gamma$ does not imply convexity of $v$ and vice versa. Clearly, when $p=e, \Gamma$ is socially stable if and only if $v$ satisfies balancedness. However, balancedness of $v$ does not imply convexity. The following example shows that convexity of $v$ does not imply social stability of $\Gamma$.

Example 4.4. Take $N=\{1,2,3\}, v(1)=v(3)=v(1,3)=0, v(2)=v(1,2)=v(2,3)=$ $v(1,2,3)=1$. Take any power vector function $p$ such that $p(1,2)=(2,1,0)^{\top}$ and $p(2,3)=$ $(0,1,2)^{\top}$. This game is convex, so the socially stable core of $\Gamma=(N, v, p)$ is non-empty. The payoff vector $x=(1,0,1)^{\top}$ is socially stable, being sustained by the stable collection $\{\{1,2\},\{2,3\}\}$. However, $x$ is not feasible and thus $\Gamma=(N, v, p)$ is not socially stable. 
Theorem 4.3 states that for convex games $v$ the socially stable core is non-empty for any power function $p$. When we make joint assumptions on $p$ and $v$, it is possible to weaken the assumptions on the characteristic function $v$. We first define the notion of $\pi$-consistency.

Definition 4.5. A power function $p: \mathcal{N} \rightarrow \mathbb{R}^{n}$ is $\pi$-consistent for a permutation $\pi$ on $N$, when for all coalitions $S$ and for all players $i$ and $j$ in $S$ it holds that $\pi(i)<\pi(j)$ implies $p_{i}(S) \leqslant p_{j}(S)$.

When the power function $p$ is $\pi$-consistent, the ranking of the players determined by permutation $\pi$ is consistent with the ranking of the power of players in any coalition. We will show that the marginal vector $m^{\pi}(v)$ belongs to the socially stable core of $\Gamma=(N, v, p)$, when $v$ is permutationally convex for the permutation $\pi$ and $p$ is $\pi$-consistent.

Theorem 4.6. Consider a structured $T U$-game $\Gamma=(N, v, p)$, where, for some permutation $\pi$ on $N, v$ is permutationally convex and $p$ is $\pi$-consistent. Then the socially stable core of $\Gamma$ contains the vector $m^{\pi}(v)$ as an element.

Proof. As in the proof of Theorem 4.3 it can be constructively shown that the collection $\left\{\pi^{i} \mid\right.$ $i \in N\}$ is socially stable. Notice that some of the weights might be zero, so the collection is not necessarily minimal. Take the payoff vector $x$ equal to the marginal vector $m^{\pi}(v)$. Then it follows for any $i \in N$ that

$$
\sum_{j \in \pi^{i}} x_{j}=\sum_{j=1}^{i}\left(v\left(\pi^{j}\right)-v\left(\pi^{j} \backslash\{j\}\right)\right)=v\left(\pi^{i}\right) .
$$

It follows that $x$ is socially stable. Moreover, since $v$ is permutationally convex for the permutation $\pi$, it follows from Granot and Huberman (1982) that $x \in C(v)$. Hence $x \in S C(v, p)$.

Under the conditions of Theorem 4.6, the socially stable core may contain multiple elements. Clearly, when $p=e$ the power function is $\pi$-consistent for any $\pi$ and $S C(v, p)=\widetilde{C}(v)$. However, when the power function $p$ is such that for some permutation $\pi$ a player $i$ has little power in any coalition involving players from $N \backslash \pi^{i}$, the socially stable core can be shown to consist of a unique element given by the marginal vector $m^{\pi}(v)$. To make this statement more precise, we introduce the notion of $\pi$-compatibility.

Definition 4.7. A power function $p: \mathcal{N} \rightarrow \mathbb{R}^{n}$ is $\pi$-compatible for a permutation $\pi$ of $N$, when for all players $i \in N$ and for all coalitions $S$ containing $i$ such that $S \backslash \pi^{i} \neq \emptyset$, it holds that $p_{i}(S)<\sum_{j=1}^{n} p_{j}(S) / n$.

When a power function is $\pi$-compatible, the power of a player $i$ in any coalition that involves another player that is ranked higher according to $\pi$, is less than the average power $\sum_{j=1}^{n} p_{j}(S) / n$. Notice that neither $\pi$-compatibility implies $\pi$-consistency nor $\pi$-consistency implies $\pi$-compatibility.

Theorem 4.8. Consider a structured $T U$-game $\Gamma=(N, v, p)$, where for some permutation $\pi$ of $N, v$ is permutationally convex and $p$ is $\pi$-compatible. Then the socially stable core of $\Gamma$ contains the marginal vector $m^{\pi}(v)$ as its unique element. 
Proof. Without loss of generality, we may normalize power functions such that $\sum_{j=1}^{n} p_{j}(S)=n$ and we may assume that the permutation $\pi$ corresponds to the ordering $\pi(i)=n+1-i, i=$ $1, \ldots, n$. Let the payoff vector $x$ belong to $S C(v, p)$ and let $\left\{S_{1}, \ldots, S_{m}\right\}$ be a stable collection of coalitions with $\left(\lambda_{1}, \ldots, \lambda_{m}\right)$ a vector of nonnegative weights such that $\sum_{j=1}^{m} \lambda_{j} p\left(S_{j}\right)=e^{N}$ and $x\left(S_{j}\right) \leqslant v\left(S_{j}\right)$ for $j=1, \ldots, m$. Notice that $\sum_{j=1}^{m} \lambda_{j}=1$.

We define the ordering $\prec^{\ell}$ on $\mathcal{N}$ by $S \prec^{\ell} T$ if and only if the lowest ranked individual in $S \cup T$ not in $S \cap T$ belongs to $S$. Without loss of generality, we may choose $\left\{S_{1}, \ldots, S_{m}\right\}$ to be minimal, and we can order the coalitions such that $S_{j} \prec^{\ell} S_{j+1}$.

We claim that, for $i=1, \ldots, m$, this stable collection satisfies $i \in S_{i} \subset\{i, \ldots, n\}$. Suppose that $\left\{S_{1}, \ldots, S_{m}\right\}$ does not contain the singleton coalition $\{n\}$. Since $p$ is $\pi$-compatible, it follows that $p_{n}\left(S_{j}\right)<1, j=1, \ldots, m$, so

$$
1=\sum_{j=1}^{m} \lambda_{j} p_{n}\left(S_{j}\right)<1,
$$

a contradiction. Consequently, $\left\{S_{1}, \ldots, S_{m}\right\}$ does contain the singleton coalition $\{n\}$, and by the properties of $\prec^{\ell}$ it follows that $S_{m}=\{n\}$.

We now use an induction argument to proceed. Assume it is true that, for some $k^{\prime} \leqslant m$, for $k=1, \ldots, k^{\prime}, n-k \in S_{m-k} \subset\{n-k, \ldots, n\}$. We show that then $n-k-1 \in S_{m-k-1} \subset$ $\{n-k-1, \ldots, n\}$. Obviously, it is not the case that $S_{m-k-1} \subset\{n-k, \ldots, n\}$ as this would violate the minimality of $\left\{S_{1}, \ldots, S_{m}\right\}$. Suppose it is not true that $S_{m-k-1} \subset\{n-k-1, \ldots, n\}$, so the lowest ranked player in $S_{m-k-1}$ is $i^{\prime}<n-k-1$. For all $S_{j}$, it holds that $p_{n-k-1}\left(S_{j}\right)<1$, so $1=\sum_{j=1}^{m} \lambda_{j} p_{n-k-1}\left(S_{j}\right)<1$, a contradiction. It follows that the lowest ranked player in $S_{m-k-1}$ is $n-k-1$. This completes the proof of the induction step, and it follows as a corollary that $m=n$.

It remains to be shown that $x=m^{\pi}(v)$. We denote $m^{\pi}(v)$ by $y$.

Obviously, it holds that $x_{n}=y_{n}=v(\{n\})$. We proceed with an induction argument. Assume it is true that, for some $k^{\prime}, x_{i}=y_{i}$ for $i=n-k^{\prime}, \ldots, n$, and $\sum_{i=n-k^{\prime}}^{n} x_{i}=v\left(\left\{n-k^{\prime}, \ldots, n\right\}\right)$. We will show that $x_{n-k^{\prime}-1}=y_{n-k^{\prime}-1}$.

Since $x$ is economically stable, it follows that $\sum_{i=n-k^{\prime}-1}^{n} x_{i} \geqslant v\left(\pi^{k^{\prime}+1}\right)$. From the induction hypothesis that $y_{i}=x_{i}$ for $i=n-k^{\prime}, \ldots, n$, we then obtain $x_{n-k^{\prime}-1} \geqslant y_{n-k^{\prime}-1}$. Since $y$ is economically stable, it follows that $\sum_{i \in S_{n-k^{\prime}-1}} y_{i} \geqslant v\left(S_{n-k^{\prime}-1}\right)=\sum_{i \in S_{n-k^{\prime}-1}} x_{i}$. Since $n-k^{\prime}-1 \in$ $S_{n-k^{\prime}-1} \subset \pi^{k^{\prime}+1}$, it follows that $x_{n-k^{\prime}-1} \leqslant y_{n-k^{\prime}-1}$. We have shown that $x_{n-k^{\prime}-1}=y_{n-k^{\prime}-1}$.

When the power function $p$ is $\pi$-compatible, any player has so much power compared to his lower-ranked players that he is able to extract all payoffs from them, up to the point where the lower-ranked players could form a deviating coalition. The socially stable core consists of a unique element, corresponding to the marginal vector $m^{\pi}(v)$. We come back to this property in the next sections.

In general, the socially stable core consists of a finite number of faces of $\widetilde{C}(v)$. To show this, for a collection of coalitions $\mathcal{F} \subset \mathcal{N}$, define

$$
C^{\mathcal{F}}(v)=\{x \in \widetilde{C}(v) \mid x(S)=v(S) \text { for all } S \in \mathcal{F}\},
$$

i.e., $C^{\mathcal{F}}(v)$ is a (possibly empty) face of $\widetilde{C}(v)$. Clearly, when $x \in S C(v, p)$ lies in the relative interior of $C^{\mathcal{F}}(v)$, then due to the linearity of the constraints every point of the face $C^{\mathcal{F}}(v)$ belongs to $S C(v, p)$. The next example shows a socially stable core consisting of two disjoint faces. 
Example 4.9. Take $N=\{1,2,3\}, v(1)=v(2)=v(3)=0, v(1,2)=4, v(1,3)=1, v(2,3)=3$, and $v(1,2,3)=6$. Since the game is superadditive, $\widetilde{C}(v)=C(v)=\left\{x \in \mathbb{R}^{3} \mid x_{1}+x_{2}+\right.$ $\left.x_{3}=6,0 \leqslant x_{1} \leqslant 3,0 \leqslant x_{2} \leqslant 5,0 \leqslant x_{3} \leqslant 2\right\}$. Take $p(1,2)=(3,1,0)^{\top}, p(1,3)=(2,0,1)^{\top}$, $p(2,3)=(0,1,3)^{\top}$, and $p(1,2,3)=(2,3,1)^{\top}$. For this power function the socially stable core $S C(v, p)$ consists of two zero-dimensional faces of the core, one being the vertex $(3,1,2)^{\top}$ and the other one being the marginal vector $(1,5,0)^{\top}$. The vector $(3,1,2)^{\top}$ is sustained by the stable collection $B_{1}=\{\{1,2,3\},\{1,2\},\{2,3\}\}$ and the vector $(1,5,0)^{\top}$ by the stable collection $B_{2}=\{\{1,2,3\},\{1,3\},\{3\}\}$.

The example is generic in the sense that the socially stable core consists of zero-dimensional faces of $\widetilde{C}(v)$ and thus consists of a finite number of payoff vectors. To prove that the finiteness property is generic indeed, observe that once the number of players is fixed, a structured TUgame is completely determined by the tuple of payoffs $v$, which can be represented by a vector in $\mathbb{R}^{2^{n}-1}$, and the tuple of power functions, which can be represented by a vector in $S^{\left(2^{n}-1\right) n}$, the unit simplex in $\mathbb{R}^{\left(2^{n}-1\right) n}$. The Euclidean topology and Lebesgue measure on $\mathbb{R}^{2^{n}-1} \times S^{\left(2^{n}-1\right) n}$ therefore induce a topology and a measure on structured TU-games.

Theorem 4.10. Let $N$ be the set of players. Then there is an open set of payoffs and power functions with full Lebesgue measure $V \times P$ such that for any $(v, p) \in V \times P$, the socially stable core of the structured TU-game $\Gamma=(N, v, p)$ is either empty or consists of a finite number of elements.

Proof. Define the closed subset $W$ of $\mathbb{R}^{n}$ with measure zero by

$$
W=\bigcup_{\left(S_{1}, \ldots, S_{n-1}\right) \in \mathcal{N}^{n-1}} \operatorname{span}\left[e^{S_{1}}, \ldots, e^{S_{n-1}}\right] .
$$

Next we define the open subset $V$ of $\mathbb{R}^{2^{n}-1}$ with full measure by

$$
V=\left\{v \in \mathbb{R}^{2^{n}-1} \mid \forall\left(S_{1}, \ldots, S_{n}\right) \in \mathcal{N}^{n} \text { with } S_{j} \neq S_{j^{\prime}} \text { when } j \neq j^{\prime},\left(v_{S_{1}}, \ldots, v_{S_{n}}\right) \notin W\right\} .
$$

Finally, we define the open subset $P$ of $S^{\left(2^{n}-1\right) n}$ with full measure as the set of vectors $p=$ $(p(S))_{S \in \mathcal{N}}$ for which it holds that any selection of $n$ vectors from the vectors $p(S), S \in \mathcal{N}$, and $e^{N}$ yields an independent set of vectors.

We now examine the socially stable core for the structured TU-game $\Gamma=(N, v, p)$, where $(v, p) \in V \times P$. All socially stable core elements are obtained by considering, for all minimal stable collections of coalitions $\left\{S_{1}, \ldots, S_{m}\right\}$, the solutions to the system of equations

$$
x\left(S_{j}\right)=v\left(S_{j}\right), \quad j=1, \ldots, m .
$$

In fact, the union over all stable collections of solutions to the corresponding system is a superset of the socially stable core. Since $\left\{S_{1}, \ldots, S_{m}\right\}$ is a stable collection, there exists a vector of nonnegative weights $\lambda$ such that

$$
\sum_{j=1}^{m} \lambda_{j} p\left(S_{j}\right)=e^{N}
$$

Moreover, $\left\{S_{1}, \ldots, S_{m}\right\}$ is minimal, so that the vectors $p\left(S_{j}\right)$ are independent, and in particular $m \leqslant n$. Since $p \in P$, it holds that $m=n$. 
Consider the system of equations,

$$
x\left(S_{j}\right)=v\left(S_{j}\right), \quad j=1, \ldots, n .
$$

If the vectors $e^{S_{j}}, j \in N$, are all independent, it follows that this system has exactly one solution, and therefore we obtain at most one socially stable core element. When the vectors $e^{S_{j}}, j \in N$, are not all independent, it follows from the definition of $V$ that this system of equations has no solution. Hence, there is at most one solution for each minimal stable collection. Since the number of minimal stable collections is finite, this proves the theorem.

Theorem 4.10 shows that in general the socially stable core refines the superadditive cover core to a great extent. There is typically only a finite number of payoff vectors in the socially stable core.

\section{Structured hierarchy games}

A hierarchical structure is a widespread organizational form. A hierarchy on $N$ is represented by a tree on $N$. A tree is a directed graph on $N$ such that there is one top-player, say player 1 , and for each player $i \neq 1$ there is a unique path of directed edges $\left(i_{1}, i_{2}\right), \ldots,\left(i_{j-1}, i_{j}\right)$ such that $i_{1}=1$ and $i_{j}=i$. Following Myerson (1977), we assume that players cannot cooperate when they are not connected. This yields the so-called restricted game $v^{r}$ assigning to any coalition $S$ the sum of the worths of the components of $S$ in the graph, i.e.,

$$
v^{r}(S)=\sum_{T \in C(S)} v(T)
$$

where $C(S)$ is the collection of maximally connected subsets of $S$ in the graph. In a hierarchy graph, a subset $S$ of nodes is connected if and only if there is a unique player $j \in S$ such that for any other player $i \in S$ there is a unique path of directed edges in the graph from $j$ to $i$ and all players on each of these paths are also in $S$. From Kaneko and Wooders (1982) and Le Breton et al. (1992) it follows that $C\left(\tilde{v}^{r}\right)$ is not empty, i.e., the core of the restricted game of the superadditive cover of $v$ is not empty, see also Demange (1994), who shows that $C\left(v^{r}\right)$ is not empty if $v$ is superadditive. From this Demange (2004) argues that hierarchies yield stability, providing a rationale for the fact that a group organizes itself in hierarchies so as to achieve coordination.

In this section we consider structured hierarchy games. In such games the social structure on the set $N$ of agents is given by a hierarchy on $N$ and the social structure on any subset $S$ is induced by this hierarchy. The characteristic function reflects the hierarchy, in the sense that $v=v^{r}$. The power function reflects the hierarchy in the sense that a player has a higher power than any of its subordinates. To be more precise, for $i \in N$, let $D(i)$ denote the set of subordinates of player $i$, i.e., for each $j \in D(i)$ there is a unique directed path from $i$ to $j$. Then the power vector for the whole set of players is a vector $p(N)$ such that for each $i$ and $j$ it holds that $p_{i}(N)>p_{j}(N)$ whenever $j \in D(i)$, i.e., the top player 1 has the highest power and the power is strictly decreasing when descending in the hierarchy. A natural example is $p_{i}(N)=|D(i)|+1$ for all $i \in N$, i.e., the power of a player is equal to the number of its subordinates including itself. Further, for any $S \subset N$ and $i \in S$ we take $p_{i}(S)=p_{i}(N)$, although it is sufficient to assume that $p_{i}(S)>p_{j}(S)$ whenever $j \in D(i)$ and $i \in S$. We now have the following theorem. 
Theorem 5.1. Consider a structured hierarchy $T U$-game $\Gamma=(N, v, p)$ where $v$ is superadditive. Then the socially stable core of $\Gamma$ contains the vector $x^{*}$, with

$$
x_{j}^{*}=v(D(j) \cup\{j\})-v(D(j)), \quad \text { for all } j \in N,
$$

as its unique element.

Proof. Let $\pi$ be any permutation on $N$ such that for all $i$ and $j$ it holds that $\pi(j)<\pi(i)$ when $p_{j}(N)<p_{i}(N)$. Clearly, the collection $\left\{\pi^{1}, \ldots, \pi^{n}\right\}$ is stable. In Demange (2004) it has been shown that $m^{\pi}(v) \in C(v)$. Since $v=v^{r}$, we have that $x^{*}=m^{\pi}(v)$, being sustained by the stable collection $\left\{\pi^{1}, \ldots, \pi^{n}\right\}$. Therefore $x^{*}$ is an element of the socially stable core. So, it remains to be proven that $x^{*}$ is the unique socially stable element of the core for any power function $p$ reflecting the hierarchy. Let $x$ be an arbitrary element of the socially stable core and let $B=$ $\left\{S_{1}, \ldots, S_{m}\right\}$ be a stable collection sustaining $x$. For $i \neq 1$, let $b(i)$ be the unique predecessor of $i$ in the hierarchy and define $b(1)=0$. Recall that player 1 is the topman of the hierarchy. Since $p_{b(i)}(S)>p_{i}(S)$ for any $S$ with $b(i) \in S$, for every player $i \in N$ it must hold that there exists an index $h(i) \in\{1, \ldots, m\}$ such that $i \in S_{h(i)}$ and $b(i) \notin S_{h(i)}$. Since $D(i)$ is the number of subordinates of player $i \in N$, we have that $|D(i)|=0$ when $i$ is a leave (being a node without subordinates), $|D(1)|=n-1$, and $|D(j)|>|D(i)|$ for all $j$ and $i \in D(j)$. We proceed now by induction on $|D(i)|$. Let node $i$ be a leave, i.e., $D(i)=\emptyset$. Notice that in a hierarchy such a node always exists. Then $\{i\}$ is a component of $S_{h(i)}$ in the graph, since $b(i) \notin S_{h(i)}$. From (i) $x$ is sustained by $S_{h(i)}$, (ii) $x$ is in the core and (iii) $v=v^{r}$ it follows that $x_{i}=v(i)$, and thus $x_{i}=x_{i}^{*}$ when $|D(i)|=0$.

Now, let $x_{i}=x_{i}^{*}$ for any player $i \in N$ with $|D(i)| \leqslant k$ for some $k, 0 \leqslant k<n-1$. When there is no player $j \in N$ with $|D(j)|=k+1$, then $x_{i}=x_{i}^{*}$ also holds for any player $i \in N$ with $|D(i)| \leqslant k+1$. Otherwise, let $j \in N$ be any player with $|D(j)|=k+1$. Since $S_{h(j)}$ sustains $x$ and $x$ is in the core, we have that $x\left(S_{h(j)}\right)=v\left(S_{h(j)}\right)$. Since $v=v^{r}$, it holds that $v\left(S_{h(j)}\right)$ is equal to the sum of the values of all the components of $S_{h(j)}$ in the graph. Together with the fact that $x$ is in the core this implies that $x(S)=v(S)$ for any component $S$ in the graph of $S_{h(j)}$. Let $C(j)$ be the component of $S_{h(j)}$ in the graph containing $j$. For any $i \in N$, define $D^{0}(i)=D(i) \cup\{i\}$. Since $b(j) \notin S_{h(j)}$ we have that $C(j) \subset D^{0}(j)$. Moreover, because of the tree structure and the fact that $j \in C(j)$, we have that $S_{h(j)} \backslash C(j)$ is partitioned in a collection of components $\left\{D^{0}\left(i_{1}\right), \ldots, D^{0}\left(i_{l}\right)\right\}$ with $i_{s} \in D(j)$ for all $s=1, \ldots, l$. Because of the induction hypothesis we have that $x\left(D^{0}\left(i_{s}\right)\right)=x^{*}\left(D^{0}\left(i_{s}\right)\right)=v\left(D^{0}\left(i_{s}\right)\right)$ for all $s$. Hence,

$$
\begin{aligned}
x\left(D^{0}(j)\right) & =x(C(j))+\sum_{s=1}^{l} x\left(D^{0}\left(i_{s}\right)\right)=v(C(j))+\sum_{s=1}^{l} v\left(D^{0}\left(i_{s}\right)\right) \\
& \leqslant v\left(D^{0}(j)\right) \leqslant x\left(D^{0}(j)\right),
\end{aligned}
$$

where the first inequality follows from the superadditivity of $v$ and the latter inequality from the fact that $x$ is in the core. Hence $x\left(D^{0}(j)\right)=v\left(D^{0}(j)\right)$.

To complete the proof, observe that $D(j)$ is partitioned in the graph in a collection of components $\left\{D^{0}\left(j_{1}\right), \ldots, D^{0}\left(j_{k}\right)\right\}$, where $\left\{j_{1}, \ldots, j_{k}\right\}$ is the set of followers of $j$. From the induction hypothesis we have that $x\left(D^{0}\left(j_{s}\right)\right)=x^{*}\left(D^{0}\left(j_{s}\right)\right)=v\left(D^{0}\left(j_{s}\right)\right)$ for all $s$. It then follows that

$$
\begin{aligned}
x_{j} & =x\left(D^{0}(j)\right)-\sum_{s=1}^{k} x\left(D\left(j_{s}\right)\right)=v\left(D^{0}(j)\right)-\sum_{s=1}^{k} v\left(D^{0}\left(j_{s}\right)\right) \\
& =v(D(j) \cup\{j\})-v(D(j))=x_{j}^{*},
\end{aligned}
$$


where the second last equality comes from the fact that $v=v^{r}$. This proves the induction step. Since the number of players is finite this completes the proof.

We remark that the permutation $\pi$ for which $x^{*}=m^{\pi}(v)$ is in general not unique. Every permutation $\pi$ that satisfies the condition in the proof yields $x^{*}$ as marginal vector. Therefore, superadditivity can be relaxed to permutational convexity of $v$ for any such permutation. Moreover, every system of power vectors $p(S)$ satisfying $p_{i}(S)>p_{j}(S)$ whenever $j \in D(i)$, yields the same socially stable core, consisting of the unique element $x^{*}$.

The outcome $x^{*}$ is very natural. Every individual receives a payoff equal to what he is contributing when he joins his subordinates. The core typically also contains other elements than the vector $x^{*}=m^{\pi}(v)$. In particular, following Demange (2004), we can replace the hierarchy graph by a communication graph by replacing all directed edges by undirected edges. Then we can choose arbitrarily some other player and reconstruct a new directed graph with this player as the top-player. Also the marginal vector with respect to a permutation reflecting this new directed graph is in the core of the game. So, for a given topman, the socially stable core with respect to a power function reflecting the corresponding hierarchy reduces the core containing several marginal vectors to one marginal vector. In the next section we consider some applications in which the hierarchy is a linear order.

\section{Applications}

\subsection{Sequencing games}

A one-machine sequencing situation, see e.g. Curiel (1988) or Hamers (1995), is described as a triple $(N, q, c)$, where $N=\{1, \ldots, n\}$ is the set of jobs in a queue to be processed, $q \in \mathbb{R}_{+}^{n}$ is an $n$-vector with $q_{i}$ the processing time of job $i$ and $c=\left(c_{i}\right)_{i \in N}$ is a collection of cost functions $c_{i}: \mathbb{R}_{+} \rightarrow \mathbb{R}_{+}$, specifying the costs $c_{i}(t)$ when $t$ is the total time needed to complete job $i$. For an ordering $\rho$ on $N$ describing the positions of the jobs in the queue, the completion time of job $i$ is given by $T_{i}(\rho)=\sum_{\{j \mid \rho(j) \leqslant \rho(i)\}} q_{j}$, i.e., the completion time is the sum of its waiting time and its own processing time, and the costs of processing $i$ are given by $C_{i}(\rho)=c_{i}\left(T_{i}(\rho)\right)$. The total costs of a coalition $S \subseteq N$ given an ordering $\rho$ are equal to $C_{S}(\rho)=\sum_{i \in S} C_{i}(\rho)$. In the sequel we assume without loss of generality that the initial positions of the jobs in the queue are given by the ordering $\rho^{0}$ with $\rho^{0}(i)=i$ for all $i \in N$, so that the costs of a coalition $S$ of jobs according to $\rho^{0}$ are given by $C_{S}\left(\rho^{0}\right)$.

Referring to the jobs as players, the grand coalition can obtain cost savings by rearranging the jobs among its members, so the minimal cost of the grand coalition is given by $C_{N}=\min _{\rho} C_{N}(\rho)$. Members of any other coalition $S$ can only rearrange their positions under the condition that the members of $S$ are not allowed to 'jump' over jobs outside $S$. So, an ordering $\rho$ is admissible for $S$ if for any $j \notin S$ the set of its predecessors does not change with respect to the initial situation. Then the minimal cost of $S$ is given by $C_{S}=\min _{\rho \in \mathcal{A}(S)} C_{S}(\rho)$, where $\mathcal{A}(S)$ is the set of admissible orderings for $S$. This gives the cost savings sequencing game with player set $N$ and characteristic function $v$ given by

$$
v(S)=C_{S}\left(\rho^{0}\right)-C_{S}, \quad S \subseteq N .
$$

From the fact that only coalitions of consecutive players can obtain cost savings, it follows straightforwardly that the game is superadditive and satisfies permutational convexity for the two permutations $u$ and $\ell$ on $N$ given by $u(i)=i, i=1,2, \ldots, n$, and $\ell(i)=n+1-i$, 
$i=1,2, \ldots, n$. Hence, the two corresponding marginal vectors $m^{u}(v)$ and $m^{\ell}(v)$ are in the core of the game $v$.

We now apply the results of the previous section. To do so, for $i<j$, denote the set $\{i, i+1$, $\ldots, j\}$ of consecutive players by $[i, j]$ and let $\mathcal{L}$ denote the set of all coalitions of consecutive players, i.e.,

$$
\mathcal{L}=\{T \in \mathcal{N} \mid T=[i, j], 1 \leqslant i \leqslant j \leqslant n\} .
$$

Then $\mathcal{L}$ equals the set of connected sets of nodes in the undirected line graph on $N$ with the set of edges given by $\mathcal{E}=\{(i, i+1) \mid i=1, \ldots, n-1\}$. Clearly on this graph the restricted game $v^{r}$ equals $v$. We now convert this undirected graph into a linearly ordered hierarchy by taking either player 1 or player $n$ as topman and let $p$ be a power function representing the hierarchy. In case player 1 is the topman, we have for every $S \in \mathcal{N}$ that

$$
p_{i}(S)<p_{j}(S) \quad \text { when } i>j ; i, j \in S,
$$

and in case player $n$ is the topman we have that

$$
p_{i}(S)<p_{j}(S) \quad \text { when } i<j ; i, j \in S .
$$

The next result follows immediately from Theorem 5.1.

Corollary 6.1. For any sequencing game it holds:

(i) $m^{\ell}(v)$ is the unique element of $S C(v, p)$ when $p$ satisfies condition (2).

(ii) $m^{u}(v)$ is the unique element of $S C(v, p)$ when $p$ satisfies condition (3).

In case (i) player $n$ gets its own value and player 1 is able to extract all gains from his cooperation with his subordinates. In case (ii) player 1 gets its own value and player $n$ is the topman who is able to extract all gains from cooperation.

To discuss these results further, we now consider the special case of linear costs, i.e., $c_{i}(t)=$ $\alpha_{i} t$ for all $t \geqslant 0$ with $\alpha_{i}>0$. In this case the cost savings game $v$ is convex, see e.g. Curiel (1988) or Hamers (1995). Moreover, in Fernández et al. (2005) it is shown that in the linear cost case the solution assigning the marginal vector $m^{u}(v)$ to each player is the unique solution that is in the core and satisfies the so-called property of Drop-Out Monotonicity (DOM). This property states that when one of the players leaves the queue, for each of the remaining players the net costs, i.e., the costs of waiting minus the payoffs obtained from the cost savings game, are non-increasing. In fact, the marginal vector $m^{u}(v)$ gives player $k$ payoff $v([1, k])-v([1, k-1])$ in the cost savings game, that means that the savings of cooperation between player $k$ and the players in front of $k$ are fully assigned to player $k$. When player $k$ leaves the game, the payoffs of the players in front of $k$ are not affected. For the players after $k$ the payoffs in the cost-savings game may decrease (because there is no gain anymore of cooperating with $k$ ), but the net costs decrease, because the waiting costs decrease when $k$ leaves the queue. So, when player $k$ drops out, the players in front of $k$ are not affected, while for the players after $k$ the costs decrease.

Although the DOM property seems to be very appealing and reasonable, it has the serious drawback that it does not give any incentive to a player to cooperate with its successors in the queue. To make the point more clear, consider a two player sequencing situation. Of course, nothing happens if the initial order of 1 before 2 is optimal already. So, suppose it is optimal to reverse the initial order and to place 2 in front of 1 , thereby generating a decrease of the costs. According to the payoff vector $m^{u}(v)$, this decrease is fully assigned to player 2 . Why should 
player 1 be willing to cooperate by agreeing to take the second position? On the contrary, player 1 has the power to play the noncooperative ultimatum game and to offer the first place in the queue to player 2 if player 2 is willing to give all the gains of this change to player 1 . Extending this reasoning we obtain that any player $j<n$ can decide upon whether or not to cooperate with his successors. This is modeled by the hierarchy with player one as the topman and power function satisfying condition (2). According to Corollary $6.1, m^{\ell}(v)$ is the unique element in $S C(v, p)$. The marginal vector $m^{\ell}(v)$ is economically stable and socially stable with respect to a social structure reflecting the dominance of any player $j$ over its successors in the sequence. The resulting cost rule does not satisfy DOM, but any player gets the gains of cooperating with her successors and therefore is willing to cooperate with her subordinates in the hierarchical structure resulting from the order in the queue.

\subsection{The water distribution problem}

In their paper 'Sharing a River' Ambec and Sprumont (2002) consider the problem of the optimal distribution of water to agents located along a river from upstream to downstream. Let $N=\{1, \ldots, n\}$ be the set of agents, numbered successively from upstream to downstream and let $f_{i} \geqslant 0$ be the flow of water entering the river between agent $i-1$ and $i, i=1, \ldots, n$, with $f_{1}$ the inflow to the most upstream agent 1 . Agent $i, i=1, \ldots, n$, has a quasi-linear utility function given by $u^{i}\left(x_{i}, t_{i}\right)=b^{i}\left(x_{i}\right)+t_{i}$, where $t_{i}$ is a monetary compensation to agent $i, x_{i}$ is the amount of water allocated to agent $i$, and $b^{i}: \mathbb{R}_{+} \rightarrow \mathbb{R}$ a continuous non-decreasing function yielding the benefit $b^{i}\left(x_{i}\right)$ to agent $i$ of the consumption $x_{i}$ of water. An allocation is a pair $(x, t) \in \mathbb{R}_{+}^{n} \times \mathbb{R}^{n}$ of water distribution and compensation scheme, satisfying

$$
\sum_{i=1}^{n} t_{i} \leqslant 0 \quad \text { and } \quad \sum_{i=1}^{j} x_{i} \leqslant \sum_{i=1}^{j} f_{i}, \quad j=1, \ldots, n .
$$

The first condition is a budget condition and says that the total amount of compensations is nonpositive, i.e., the compensations only redistribute the total welfare. The second condition reflects that any agent can use the water that entered upstream, but that the water inflow downstream of some agent can not be allocated to this agent. Because of the quasi-linearity and the possibility of making money transfers, an allocation is Pareto optimal if and only if the distribution of the water streams maximizes the total benefits, i.e., the water distribution $x^{*} \in \mathbb{R}_{+}^{n}$ solves the following maximization problem:

$$
\max _{x} \sum_{i=1}^{n} b^{i}\left(x_{i}\right) \quad \text { s.t. } \quad \sum_{i=1}^{j} x_{i} \leqslant \sum_{i=1}^{j} f_{i}, \quad j=1, \ldots, n .
$$

A welfare distribution allocates the total benefits of an optimal water distribution $x^{*}$ over the agents, i.e., it is a vector $z \in \mathbb{R}^{n}$ assigning utility $z_{i}$ to agent $i$ and satisfying $\sum_{i=1}^{n} z_{i}=$ $\sum_{i=1}^{n} b^{i}\left(x_{i}^{*}\right)$. Clearly, any welfare distribution can be implemented by the allocation $(x, t)$ with $x_{i}=x_{i}^{*}$ and $t_{i}=z_{i}-b^{i}\left(x_{i}^{*}\right), i=1, \ldots, n$.

The problem to find a reasonable welfare distribution can be modeled as a TU-game. Obviously, for any pair of players $i, j$ with $j>i$ it holds that the water inflow entering the river before the upstream agent $i$ can only be allocated to the downstream agent $j$ if all agents between $i$ and $j$ cooperate, otherwise any agent between $i$ and $j$ can take the flow from $i$ to $j$ for its own use. Hence, only coalitions of consecutive agents are admissible. Clearly, for $S=N$, 
$v(N)=\sum_{i=1}^{n} b^{i}\left(x_{i}^{*}\right)$ with $x^{*} \in \mathbb{R}^{n}$ the solution of maximization problem (4). For any connected coalition $S=[i, j] \in \mathcal{L}$, its worth is given by

$$
\begin{aligned}
& v([i, j])=\sum_{h=i}^{j} b^{h}\left(x_{h}^{* S}\right), \quad \text { where } x^{* S}=\left(x_{h}^{* S}\right)_{h=i}^{j} \text { solves } \\
& \max _{x_{i}, \ldots, x_{j}} \sum_{h=i}^{j} b^{h}\left(x_{h}\right) \quad \text { s.t. } \quad \sum_{k=i}^{h} x_{k} \leqslant \sum_{k=i}^{h} f_{k}, \quad h=i, \ldots, j .
\end{aligned}
$$

Without loss of generality we normalize the benefit functions by taking $b^{i}\left(f_{i}\right)=0$, implying that $v(\{i\})=b^{i}\left(f_{i}\right)=0, i=1, \ldots, n$, so that the values $v(S)$ for $|S| \geqslant 2$ represent the net gains of cooperating. Again it follows from the fact that only coalitions of consecutive players can cooperate that the game is superadditive and satisfies permutational convexity for the two permutations $u$ and $\ell$, so that $m^{u}(v)$ and $m^{\ell}(v)$ are in the core. In case all functions $b^{i}$ are differentiable with derivative going to infinity as $x_{i}$ tends to zero, strictly increasing and strictly concave, Ambec and Sprumont (2002) have shown that the game is convex and that the solution assigning the marginal vector $m^{u}(v)$ to the game is the unique solution that is in the core and satisfies a certain fairness condition. This condition (quite different from the fairness condition of Myerson to characterize the Shapley value) says that any coalition $S$ gets at most its aspiration level, being the highest utility it can obtain when it may use all the water of all the agents $1, \ldots, \hat{s}$, where $\hat{s}=\max \{s \mid s \in S\}$. Clearly, this implies that any coalition $[1, j]$ can get at most $v([1, j]), j=1, \ldots, n$, so that it trivially follows that indeed the marginal vector $m^{u}(v)$ assigning $m_{i}^{u}(v)=v([1, i])-v([1, i-1])$, $i=1, \ldots, n$, is the unique candidate in the core satisfying the aspiration requirements.

As in the sequencing situation again we have that the payoff vector $m^{u}(v)$ has the property that when a player $j$ does not want to cooperate, the players in front of $j$, including $j$ itself, are not hurt. Like in the sequencing game, this is a very counterintuitive outcome. Although any upstream coalition $[1, j]$ can prevent that coalition $[j+1, n]$ gets more than $v([j+1, n])$ by using all flows $f_{1}, \ldots, f_{j}$ by itself, all benefits from cooperation go to the coalition $[j+1, n]$. Again the outcome $m^{u}(v)$ has the serious drawback that it does not give any incentive to a player $j$ to cooperate with its successors in the queue. On the other hand, considering again a two agent situation, also in this river game player 1 has the power to play the noncooperative ultimatum game and to pass the stream $f_{1}-x_{1}^{*}$ to player 2 if this player is willing to give up all the gains of cooperation. We may argue that player 1 is in control of whether or not to cooperate by letting through a part of its water inflow $f_{1}$. In general, any player is in control of cooperation with its successors. This again can be modeled by a hierarchy consisting on the line graph with player 1 as the topman and corresponding power function satisfying condition (2). According to Corollary $6.1, m^{\ell}(v)$ is the unique element in the socially stable core, yielding an outcome reflecting to a social structure in which any player $j$ dominates its successors in the sense that player $j$ controls the water inflow up to $j$.

\section{Conclusions}

In this paper we consider structured transferable utility games. In such games each coalition is organized according to some social structure, for instance a network, a hierarchy or some other dominance relation. There exist several measures in the literature to measure the power of the players within a coalition having some social structure. In this way the social structure of a coalition can be represented by a power vector. A collection of coalitions is stable if weights 
can be assigned to these coalitions such that the total weighted power of every agent is the same. Stability generalizes the well-known concept of balancedness, where all members of a coalition have the same power. The socially stable core consists of the economically stable payoff vectors that are sustained by a stable collection of coalitions.

The socially stable core is a subset of the superadditive cover core, and therefore of the core whenever the game is superadditive. We provide several results on non-emptiness and uniqueness of the socially stable core. If the game is convex, then the socially stable core is non-empty for any social structure. Also, the socially stable core is non-empty if for some permutation the game is permutationally convex and the power function is permutationally consistent. If the game is permutationally convex and the power function is permutationally compatible, then the socially stable core consists of only one element, being the marginal vector corresponding to the permutation. Generically, the socially stable core consists of a finite number of elements only. Therefore, the socially stable core is a forceful selection device for the superadditive cover core.

We apply the socially stable core concept to structured hierarchy games. Under rather mild conditions the socially stable core of a structured hierarchy game consists only of a specific marginal vector. This vector is completely determined by the hierarchical structure on the players. The sequencing problem and the water distribution problem are modeled as two examples of structured hierarchy games. As a consequence, we are able to advocate a unique solution for these two problems.

In this paper the social structure has been given exogenously. In the network literature, the social structure is the result of a link formation process. It would be interesting to combine insights provided by this paper with those coming from the network formation literature. In this way one can both endogenize the social structure and the distribution of network payoffs, which opens up an interesting line of further research.

\section{Acknowledgment}

P. Jean-Jacques Herings would like to thank the Netherlands Organization for Scientific Research (NWO) for financial support.

\section{References}

Ambec, S., Sprumont, Y., 2002. Sharing a river. J. Econ. Theory 107, 453-462.

Aumann, R.J., Drèze, J.H., 1974. Cooperative games with coalition structure. Int. J. Game Theory 3, $217-237$.

Aumann, R.J., Maschler, M., 1964. The bargaining set for cooperative games. In: Dresher, M., Shapley, S., Tucker, A.W. (Eds.), Advances in Game Theory. In: Ann. of Math. Stud., vol. 52. Princeton Univ. Press, Princeton, pp. $443-476$.

Bondareva, O.N., 1963. Some applications of linear programming methods to the theory of cooperative games. Problemy Kibernetiki 10, 119-139 (in Russian).

Borm, P., Owen, G., Tijs, S., 1992. On the position value for communication situations. SIAM J. Discrete Math. 5, $305-320$.

Curiel, I., 1988. Cooperative game theory and applications. PhD thesis. Katholieke Universiteit Nijmegen.

Demange, G., 1994. Intermediate preferences and stable coalition structures. J. Math. Econ. 23, 45-58.

Demange, G., 2004. On group stability in hierarchies and networks. J. Polit. Economy 112, 754-778.

Faigle, U., Kern, W., 1992. The Shapley value for cooperative games under precedence constraints. Int. J. Game Theory $21,249-266$.

Fernández, C., Borm, P., Hendrickx, R., Tijs, S., 2005. Drop-out monotonicity rules for sequencing games. Math. Methods Operations Res. 61, 501-504.

Friedman, J.W., 1989. Game Theory with Applications to Economics. Oxford Univ. Press, New York.

Gilles, R.P., Owen, G., van den Brink, R., 1992. Games with permission structures: The conjunctive approach. Int. J. Game Theory 20, 277-293. 
Granot, G., Huberman, G., 1982. The relationship between convex games and minimal cost spanning tree games: a case for permutationally convex games. SIAM J. Algebraic Discrete Methods 3, 288-292.

Hamers, H., 1995. Sequencing and delivery situations: A game theoretic approach. PhD thesis. Tilburg University.

Harsanyi, J.C., 1959. A bargaining model for cooperative $n$-person games. In: Tucker, A.W., Luce, R.D. (Eds.), Contributions to the Theory of Games, vol. IV. Princeton Univ. Press, Princeton, pp. 325-355.

Hart, S., Kurz, M., 1983. Endogenous formation of coalitions. Econometrica 51, 1047-1064.

Herings, P.J.J., van der Laan, G., Talman, A.J.J., 2003. Socially structured games and their applications. Research memorandum 03/09. METEOR, Maastricht University.

Herings, P.J.J., van der Laan, G., Talman, A.J.J., 2005. The positional power of nodes in digraphs. Soc. Choice Welfare 25, $1-16$.

Ichiishi, T., 1981. Super-modularity: Applications to convex games and to the greedy algorithm for LP. J. Econ. Theory $25,283-286$.

Jackson, M.O., Wolinsky, A., 1996. A strategic model of social and economic networks. J. Econ. Theory 71, 44-74.

Kaneko, M., Wooders, M.H., 1982. Cores of partitioning games. Math. Soc. Sci. 3, 313-327.

Kalai, E., Postlewaite, A., Roberts, J., 1978. Barriers to trade and disadvantageous middlemen: Nonmonotonicity of the core. J. Econ. Theory 19, 200-209.

Le Breton, M., Owen, G., Weber, S., 1992. Strongly balanced cooperative games. Int. J. Game Theory 20, 419-427.

Myerson, R.B., 1977. Graphs and cooperation in games. Math. Operations Res. 2, 225-229.

Owen, G., 1977. Values of games with a priori unions. In: Henn, R., Moeschlin, O. (Eds.), Essays in Mathematical Economics and Game Theory. Springer-Verlag, Berlin, pp. 76-88.

Owen, G., 1986. Values of graph-restricted games. SIAM J. Algebraic Discrete Methods 7, 210-220.

Owen, G., 1995. Game Theory. Academic Press, San Diego.

Piccione, M., Rubinstein, A., 2006. Equilibrium in the jungle. Working paper.

Rubenstein, A., 1980. Ranking the participants in a tournament. SIAM J. Appl. Math. 38, 108-111.

Shapley, L.S., 1953. A value for $n$-person games. In: Kuhn, H.W., Tucker, A.W. (Eds.), Contributions to the Theory of Games, vol. II. Princeton Univ. Press, Princeton, pp. 307-317.

van den Brink, R., Gilles, R.P., 1996. Axiomatizations of the conjunctive permission value for games with permission structures. Games Econ. Behav. 12, 113-126.

van den Brink, R., Gilles, R.P., 2000. Measuring combination in directed networks. Soc. Networks 22, 141-157.

Winter, E., 1989. A value for cooperative games with levels structure of cooperation. Int. J. Game Theory 18, 227-240. 